\title{
REVISED Any neuron can perform linearly non-separable
}

\section{computations [version 2; peer review: 1 approved, 1 approved with reservations]}

\author{
Romain D. Cazé(iD \\ CNRS IEMN UMR 8520, Villeneuve d'ascq, Haut de France, 59650, France
}

V2 First published: 06 Jul 2021, 10:539

https://doi.org/10.12688/f1000research.53961.1

Second version: 16 Sep 2021, 10:539

https://doi.org/10.12688/f1000research.53961.2

Latest published: 08 Jun 2022, 10:539

https://doi.org/10.12688/f1000research.53961.3

\section{Abstract}

Multiple studies have shown how dendrites enable some neurons to perform linearly non-separable computations. These works focus on cells with an extended dendritic arbor where voltage can vary independently, turning dendritic branches into local non-linear subunits. However, these studies leave a large fraction of the nervous system unexplored. Many neurons, e.g. granule cells, have modest dendritic trees and are electrically compact. It is impossible to decompose them into multiple independent subunits. Here, we upgraded the integrate and fire neuron to account for saturating dendrites. This artificial neuron has a unique membrane voltage and can be seen as a single layer. We present a class of linearly nonseparable computations and how our neuron can perform them. We thus demonstrate that even a single layer neuron with dendrites has more computational capacity than without. Because any neuron has one or more layer, and all dendrites do saturate, we show that any dendrited neuron can implement linearly non-separable computations.

Keywords

Dendrites, computation, linearly non-separable, neuroscience

\section{“incf}

This article is included in the INCF gateway.

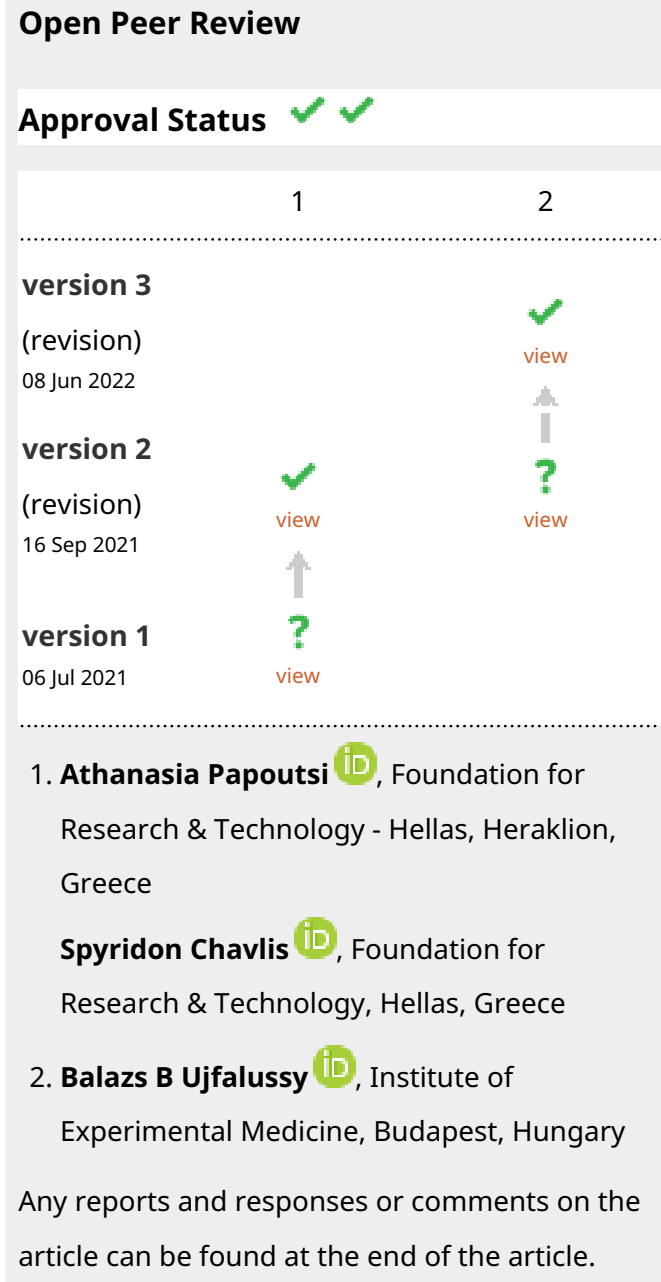


Corresponding author: Romain D. Cazé (romain.caze@protonmail.com)

Author roles: Cazé RD: Conceptualization, Formal Analysis, Investigation, Methodology, Software, Validation, Writing - Original Draft Preparation, Writing - Review \& Editing

Competing interests: No competing interests were disclosed.

Grant information: This work was supported by the Centre National de la Recherche Scientifique [ANR-UWAKE]. The funders had no role in study design, data collection and analysis, decision to publish, or preparation of the manuscript.

Copyright: ๑ 2021 Cazé RD. This is an open access article distributed under the terms of the Creative Commons Attribution License, which permits unrestricted use, distribution, and reproduction in any medium, provided the original work is properly cited.

How to cite this article: Cazé RD. Any neuron can perform linearly non-separable computations [version 2; peer review: 1 approved, 1 approved with reservations] F1000Research 2021, 10:539 https://doi.org/10.12688/f1000research.53961.2

First published: $06 \mathrm{Jul}$ 2021, 10:539 https://doi.org/10.12688/f1000research.53961.1 


\section{REVISED Amendments from Version 1}

We do not use a reduction in driving force to implement sublinear summation. We added a paragraph to the discussion to make this point crystal clear. We updated the code and upgraded the figure to change time->episode. Fig.1C demonstrates what we mean by an episode. We added a reference to the figure in the result paragraph. Finally we made slight changes to take into account all the minor comments from the reviewer.

Any further responses from the reviewers can be found at the end of the article

\section{Introduction}

We show here how dendrites can extend the computational capacity of all neurons, even the tiniest. We already knew that dendrites might extend the computational capacity of some pyramidal neurons. Their extended dendrites capable of dendritic spikes changed the way we saw them $\left(\operatorname{see}^{2}\right.$ for one of the first articles presenting this idea). More recently a study suggested that we should model these neurons as a two layer neural networks. ${ }^{6}$ This theoretical model was further consolidated by experiments showing that we can see a pyramidal neuron as a collection of non-linear subunits. ${ }^{7}$ Certain non-linearities can even allow a dendrite to implement the exclusive or (XOR). ${ }^{9}$ Moreover, a similar kind of nonmonotonic non-linearity was found in human pyramidal neurons. ${ }^{4}$ But what about other neurons with modest dendrites incapable of spiking?

Pyramidal neurons only represent a fraction of all neurons. For instance, the dendrites of cerebellar stellate cells cannot emit spikes, but they do saturate ${ }^{1}$ and they can be decomposed into multiple independent subunits - with independent membrane voltages - turning them into two-stage units like the pyramidal neuron. ${ }^{8}$ Previously we have shown that passive dendrites are sufficient to enable a neuron to perform linearly non-separable computations, for instance, the feature binding problem. ${ }^{3}$ We focus here on cells with a modest and passive dendritic tree. These cells form a single layer unit. In the present study, we demonstrate that these neurons can still implement a linearly non-separable computation. We use them as the simplest common denominator, as even spiking dendrites do saturate, and a 2 layer network can perform all the computation of a single layer architecture and more.

\section{Methods}

An integrate and fire neuron with dendrites (the DIF)

We started from a leaky integrate and fire (LIF). This model has a membrane $V$ modelled by the following equation:

$$
\tau \frac{d v}{d t}=\left(v_{E}-v(t)\right)+R I_{s}(t)
$$

With $\tau=20 \mathrm{~ms}$ the neuron time constant, $v(t)$ the membrane voltage at time $t$ and $v_{E}=-62 \mathrm{mV}$ which sets the resting membrane voltage. $R=20 \mathrm{M} \Omega$ is the value of the resistance and $I_{s}(t)$ models the time varying synaptic inputs conductance.

$$
I_{s}(t)=\sum_{i} g_{d^{i}}(t)\left(E_{s}-v(t)\right)
$$

This current depends on the difference between $v(t)$ the neuron voltage, equal everywhere, and $E_{s}$ the synaptic reversal potential $(0 \mathrm{mV})$ while $g_{d^{i}}$ is the synaptic conductance in dendrite $i$. Each $g_{d^{i}}$ is bounded between 0 and $10 p S$. Each $g_{d^{i}}$ jumps up instantaneously to its maximal value for each incoming input spike and decays exponentially with time constant $\tau_{s}=1 \mathrm{~ms}$. In a LIF all synaptic inputs are gathered into a single umbrella and $i=1$. In the present study, we introduce the Dendrited Integrate and Fire (DIF) which includes at least two dendrites $(i=2)$. We cluster synaptic inputs into two groups, each targeting a dendrite (one green and one blue, see Figure 1). We used the Brian software version 2 to carry out our simulations, the code is freely available on the git repository attached with this report. ${ }^{10}$

Boolean algebra refresher

First, let's present Boolean functions:

Definition 1. A Boolean function of $n$ variables is a function on $\{0,1\}^{n}$ into $\{0,1\}$, where $n$ is a positive integer.

Importantly, we commonly assume that neurons can only implement linearly separable computations:

Definition 2. $f$ is a linearly separable computation of $\mathrm{n}$ variables if and only if there exists at least one vector $w \in \mathcal{R}^{n}$ and a threshold $\Theta \in \mathcal{R}$ such that: 


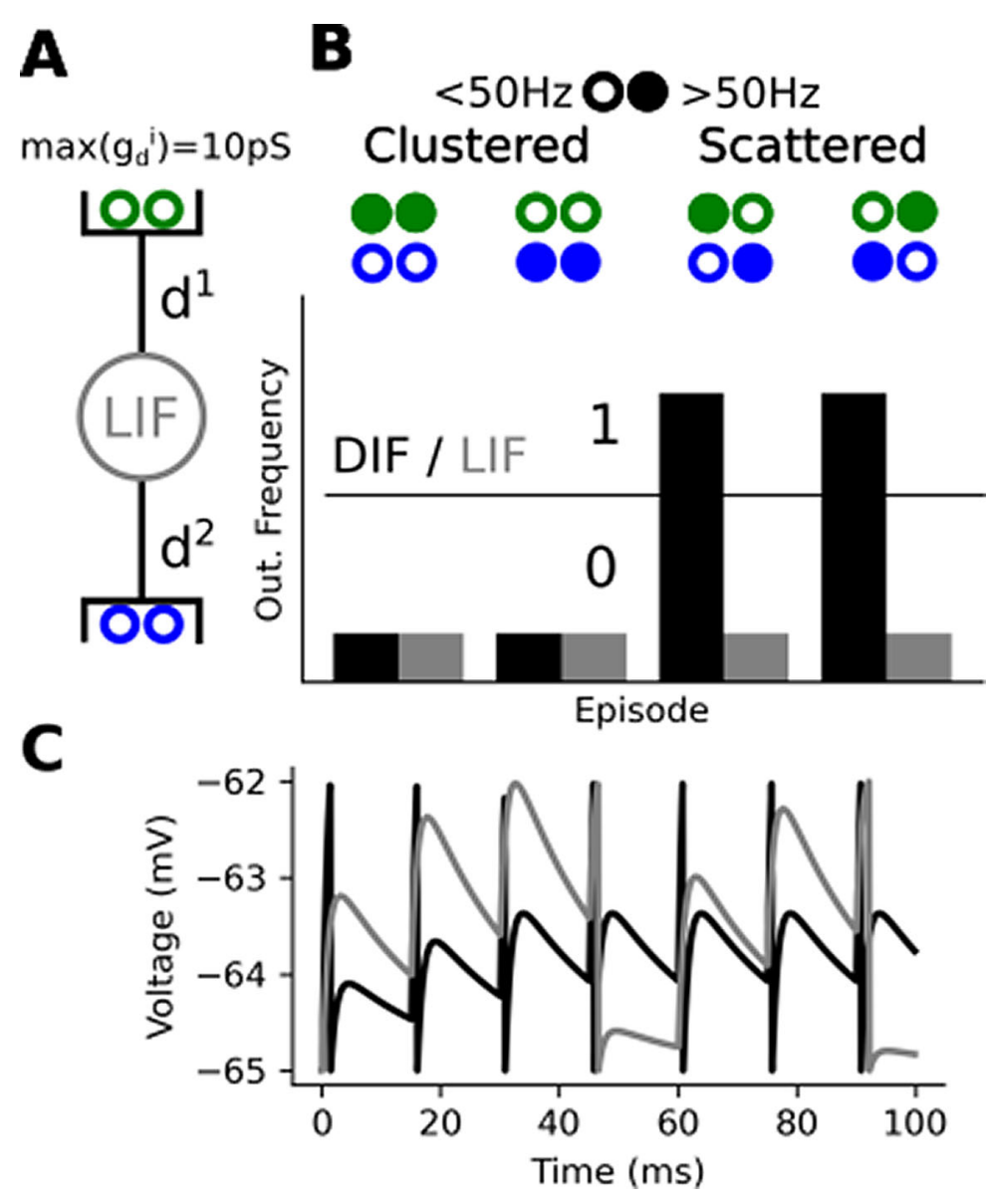

Figure 1. A dendrited integrate and fire implementing a linearly non-separable computation. (A) A leaky integrate and fire (LIF) with two dendrites making it a dendrited integrate and fire (DIF), each half of the 4 synaptic inputs targets a distinct dendrite where $g$ locally saturates at $10 p S$ (B) Four stimulation episodes, filled circles stand for $a>50 \mathrm{~Hz}$ input spike train while empty circles stand for $>50 \mathrm{~Hz}$ input spike train. Below, we plotted the response of the DIF (black) and a LIF (grey) during the episode. We purposely removed the ticks label as the frequencies depend on the parameter of the model and input regularity. The parameters of the model can vary largely without affecting the observation. (C) Some voltage response during the 3rd episode. In the clustered case (grey), the neuron reach spike threshold three times whereas it reaches spike thresold seven times in the scattered case (black). ${ }^{5}$

$$
f(X)= \begin{cases}1 & \text { if } w \cdot X \geq \Theta \\ 0 & \text { otherwise }\end{cases}
$$

where $X \in\{0,1\}^{n}$ is the vector notation for the Boolean input variables.

\section{Results}

The compact feature binding problem (cFBP)

In this section, we demonstrate a class of compact linearly inseparable (non-separable) computations that we are going to study. These computations are compact because they have four input/output lines.

We entirely specify an example in Table 1 . This computation that we call the compact feature binding problem (cFBP) is linearly inseparable.

Proposition 1. The cFBP is linearly inseparable (non-separable)

Proof. The output must be 0 for two disjoint couples $(1,2)$ and $(3,4)$ of active inputs. It means that $w_{1}+w_{2} \leq \Theta$, and $w_{3}+w_{4}$ $\leq \Theta$, and we can add these two inequalities to obtain $w_{1}+w_{2}+w_{3}+w_{4} \leq 2 \Theta$. However, the output must be 1 for two other couples made of the same active inputs $(1,3)$ and $(2,4)$. It means that $w_{1}+w_{3}>\Theta$, and $w_{2}+w_{4}>\Theta$, and we can add these 
Table 1. The truth table of a non-linearly separable computation.

\begin{tabular}{|l|l|}
\hline Inputs & Output \\
\hline 0011 & 0 \\
\hline 1100 & 0 \\
\hline 0101 & 1 \\
\hline 1010 & 1 \\
\hline
\end{tabular}

two inequalities to obtain $w_{1}+w_{2}+w_{3}+w_{4}>2 \Theta$. This yield a contradiction proving that no weights set exists solving this set of inequalities.

The cFBP is simple in two ways:

- Four input/output relations define this computation - the same number as the famous XOR (exclusive or).

- Contrary to the XOR it can be implemented with excitatory inputs and a monotone transfer function. ${ }^{3}$

We can extend the cFBP by increasing the number of inputs. In this case we deal with tuples instead of couples. As such, the cFBP corresponds to an entire family of linearly inseparable computations, and a dendrited neuron can implement them using the strategy that we will present in the next section.

A LIF with its linear integration cannot implement such a computation. While a neuron with two saturating dendrites can easily implement it. We already proved how a ball-and-stick biophysical model can implement this computation in a previous study. ${ }^{3}$

\section{Implementing the cFBP in a dendrited integrate and fire}

We use two independently saturating conductances to implement the cFBP in a minimal extension of the LIF that we called the dendrited integrated and fire (DIF). The DIF has a single membrane voltage to account for its compactness so we might wonder how local saturation can arise in such a morphology. Saturation has two possible origins: (1) a reduction in driving force can cause saturation as in, ${ }^{1}$ but (2) it can also be due to the intrinsic limitations in conductance per unit of surface. This latter possibility makes saturation possible in an electrically compact neuron. Even in a neuron with a small dendritic tree, the conductance is going to reach an upper bound per unit of surface and the only possibility to increase excitation consists in stimulating a larger area. We are going to employ this local bounding of the conductance to implement the cFBP in a DIF.

To do that, we only need two dendrites as shown in Figure 1A. We can interpret the $0 \mathrm{~s}$ and $1 \mathrm{~s}$ in the truth table in at least two ways: (1) either the pre- or post-synaptic neurons activates (2) or they reach a given spike frequency. In the following section, we will use the latter interpretation. Consequently, we consider a pre-synaptic input active when it fires above $50 \mathrm{~Hz}$ regular spike-train and inactive if it fires below $50 \mathrm{~Hz}$ (this value is arbitrary and can largely vary to match a neuron working range). We stimulate our model in four distinct episodes to reproduce the truth table from the previous section. You can observe on Figure 1 that locally bounding $g$ enables implement the of cFBP. When $g$ has no bound, the membrane voltage always reaches the spiking threshold at the same speed (LIF case). When we locally bound conductances the membrane voltage takes more time (45 ms see Figure 1C) to reach threshold in the clustered case (total $g=10 p S$ ) than in the scattered case (total $g=20 p S$ ). All in all, a DIF will respond differently for the clustered and scattered case while a LIF won't. This enables a DIF to implement the cFBP while a LIF can't.

\section{Discussion/conclusion}

In this brief report, we introduced a small extension to the leaky integrate and fire neuron: a dendrited integrate and fire neuron which can implement linearly non-separable computations. This single layer model applied to cerebellar granule cells predicts that they can implement a linearly non-separable computation. These neurons have on average four dendrites, but we have shown here that two suffice. The DIF's multiple distinctly bounded $g$ underlie this ability. For example, we need a local saturation of $g_{d^{i}}$ to implement the cFBP.

Importantly, a reduction in driving force does not generate sublinear summation in a DIF. The implementation of a linearly inseparable computation would have been impossible in a single compartment neuron because of interaction via the unique membrane potential. The usage of locally bounded $g$ is crucial to make our prediction possible. 
The experiment demonstrating this prediction seems straightforward. One would need to stimulate four distinct groups of mossy fibres following our different scenarios. We could then record how a group of granule cell respond using optogenetics reporting (i.e. calcium imaging). We predict that a significant part of granule cells might implement the cFBP. This prediction could reveal the true potential of single neurons. The next step consists of looking at the network level as already done with spiking dendrites. ${ }^{5}$

\section{Data availability}

No data are associated with this article.

\section{Software availability}

- Source code available from: https://github.com/rcaze/21_03Ca/tree/1.

- Archived source code: https://doi.org/10.5281/zenodo.5355354. ${ }^{10}$

- License: MIT license.

\section{Acknowledgements}

I used "we" as science is a collective endeavour. Discussions on this topic had begun as early as 2013 with my former PhD Advisor and collaborators from Institut Pasteur Paris. I also want to acknowledge M. Humphries, F Zeldenrust, A. Foust for their valuable comments on the early draft and Ms Marini-Audouard for the proof-reading before submission. An earlier version of this article can be found on bioRxiv (doi: https://doi.org/10.1101/2021.04.02.438177).

1. Abrahamsson T, Cathala L, Matsui K, et al.: Thin dendrites of cerebellar interneurons confer sublinear synaptic integration and a gradient of short-term plasticity. Neuron. 73(6): 1159-1172.

PubMed Abstract | Publisher Full Text

2. Bartlett M: The clusteron: towards a simple abstraction to a complex neuron. Advances in Neural Information Processing Systems.4.

3. Cazé RD, Humphries M, Gutkin B: Passive dendrites enable single neurons to compute linearly non-separable functions. PLOS Comput Biol. 9(2): e1002867. PubMed Abstract | Publisher Full Text | Free Full Text

4. Gidon A, Zolnik TA, Fidzinski $P$, et al.: Dendritic action potentials and computation in human layer $2 / 3$ cortical neurons. Science. 367(6473): 83-87. PubMed Abstract | Publisher Full Text

5. Memmesheimer R-M, Timme M: Non-additive coupling enables propagation of synchronous spiking activity in purely random networks. PLOS Comput Biol. 8(4): e1002384.

PubMed Abstract | Publisher Full Text | Free Full Text

6. Poirazi P, Brannon T, Mel BW: Pyramidal neuron as two-layer neural network. Neuron. 37(6): 989-999. PubMed Abstract | Publisher Full Text

7. Polsky A, Mel BW, Schiller J: Computational subunits in thin dendrites of pyramidal cells. Nat Neurosci. 7(6): 621-627. PubMed Abstract | Publisher Full Text

8. Tzilivaki A, Kastellakis G, Poirazi P: Challenging the point neuron dogma: FS basket cells as 2-stage nonlinear integrators. Nat Commun. 10(1): 3664.

PubMed Abstract | Publisher Full Text | Free Full Text

9. Zador AM, Claiborne BJ, Brown TH: Nonlinear pattern separation in single hippocampal neurons with active dendritic membrane. Advances in Neural Information Processing Systems. page 8.

10. Dr Romain DC: rcaze/21_03Ca: F1000 v2 (Version 2). Zenodo. 2021. Publisher Full Text 


\section{Open Peer Review}

\section{Current Peer Review Status:}

\section{Version 2}

Reviewer Report 15 February 2022

https://doi.org/10.5256/f1000research.77421.r121426

(C) 2022 Ujfalussy B. This is an open access peer review report distributed under the terms of the Creative Commons Attribution License, which permits unrestricted use, distribution, and reproduction in any medium, provided the original work is properly cited.

\section{Balazs B Ujfalussy}

Laboratory of Biological Computation, Institute of Experimental Medicine, Budapest, Hungary

In this report the author extends his previous work (ref 3.) demonstrating that simple neurons with two saturating nonlinearities can implement certain non-trivial computational problems, i.e., the feature binding problem (FBP). The novelty of the current implementation is that it places the nonlinearity to the synaptic conductance term in the input instead of to the reduction of the synaptic driving force. This way the FBP can be implemented with electrically compact neurons without the need for independent electrical subunits.

\section{I have two main concerns:}

Although the idea that nonlinear integration between different input streams could be implemented at the synaptic conductance level, actual experimental data supporting this hypothesis is not cited.

The authors introduce the Dendritic Integrate and Fire model as a variant of the Leaky Integrate and Fire harboring multiple groups of interacting synaptic conductance. Since the model does not assume that the sites target physically separate dendritic compartments, I found the name potentially misleading.

\section{Specific comments:}

The introduction states that cerebellar granule cells can be decomposed into multiple independent subunits, but the reference cited [ref. 8] does not directly imply this.

In Eq. 1. I_s is input current and not input conductance

I suggest to highlight that $g$ in Eq. 2. denotes the total synaptic conductance associated with a group of input synapses, which is quite unusual assumption for most modelers.

Fig. 1: in panel B grey and black denote the DIF and LIF models. In panel C the same colors denote clustered versus scattered configurations, according to the legend.

Fig 1C: It is unclear what were the inputs used here: As far as I understand, there are 4 spike 
trains, 2 of which the input frequency $\mathrm{F}>50 \mathrm{~Hz}$ and 2 with $\mathrm{F}<50 \mathrm{~Hz}$. What are the actual frequencies? Are these Poisson trains? Based on the response, I see a strong $60 \mathrm{~Hz}$ drive, but I don't see any other periodicity in the response. I would consider showing the timing of the inputs with green and blue ticks above the response.

Fig. 1C: It is hard to see the spikes on the response - I would consider showing a larger yaxis range.

Fig. 1C: It seems to me that the membrane potential is reset after each spike, but details of this reset are missing.

Fig 1, legend: It is not explained how reference 5 is related to the figure.

It is not clear why cFBP is compact. It is an $n=4$-dimensional problem, so it is defined by its $2^{\wedge} 4=16$ input-output pairs. Even if we restrict ourselves to the mappings with exactly 2 of the inputs being active, there are 6 of such pairs. I understand that it remains linearly nonseparable no matter how we define the remaining two mappings, but the definition ('compact because they have four input/output lines') still feels somewhat vague and arbitrary.

The statement 'a reduction in driving force does not generate sublinear summation in a DIF' is false. Reduction of driving force would generate sublinear summation even in a DIF. What the author might want to say is that in this particular example sublinear integration was not associated with reduction of driving force.

Last paragraph: It is unclear how the proposed experiment would test whether the granule cells implementing cFBP use saturating input conductance or driving force reduction as a biophysical mechanism to solve the FBP.

Is the work clearly and accurately presented and does it cite the current literature? Partly

Is the study design appropriate and is the work technically sound? Partly

Are sufficient details of methods and analysis provided to allow replication by others? Yes

If applicable, is the statistical analysis and its interpretation appropriate? Not applicable

Are all the source data underlying the results available to ensure full reproducibility? Yes

Are the conclusions drawn adequately supported by the results? Yes 
Competing Interests: No competing interests were disclosed.

Reviewer Expertise: Computational Neuroscience

I confirm that I have read this submission and believe that I have an appropriate level of expertise to confirm that it is of an acceptable scientific standard, however I have significant reservations, as outlined above.

Author Response 10 Jun 2022

Romain Cazé, CNRS UMR 8520, France

Although the idea that nonlinear integration between different input streams could be implemented at the synaptic conductance level, actual experimental data supporting this hypothesis is not cited.

- I proposed glutamate spillover as a candidate where nearby synapses interact. However, its effect on firing was never investigated to our knowledge. This work thus make a strong experimental prediction and give a computational role for glutamate spillover. I added two references to further support this argument.

The authors introduce the Dendritic Integrate and Fire model as a variant of the Leaky Integrate and Fire harboring multiple groups of interacting synaptic conductance. Since the model does not assume that the sites target physically separate dendritic compartments, I found the name potentially misleading.

- I renamed our model the Saturating Integrate and Fire, this model account for a neuron where synapses target distinct points where they interact. I now discuss this statement extensively in our conclusion.

Specific comments:

The introduction states that cerebellar granule cells can be decomposed into multiple independent subunits, but the reference cited [ref. 8] does not directly imply this.

- I precised that ref.6 concerns pyramidal neurons only and not granule cells. I also insist in the introduction on the fact that granule cells are isopotential structure impossibl to decompose into subunits.

In Eq. 1. I_s is input current and not input conductance

- I corrected the mistake

I suggest to highlight that $g$ in Eq. 2. denotes the total synaptic conductance associated with a group of input synapses, which is quite unusual assumption for most modelers.

- I now underline this crucial difference and discuss it in our conclusion 
Fig. 1: in panel B grey and black denote the DIF and LIF models. In panel C the same colors denote clustered versus scattered configurations, according to the legend.

- I redraw the panel B and C of this figure to make it clearer.

Fig 1C: It is unclear what were the inputs used here: As far as I understand, there are 4 spike trains, 2 of which the input frequency $F>50 \mathrm{~Hz}$ and 2 with $F<50 \mathrm{~Hz}$. What are the actual frequencies? Are these Poisson trains? Based on the response, I see a strong $60 \mathrm{~Hz}$ drive, but I don't see any other periodicity in the response. I would consider showing the timing of the inputs with green and blue ticks above the response.

- I now explicit this point in the figure legend.

Fig. 1C: It is hard to see the spikes on the response - I would consider showing a larger y-axis range.

- Spikes arised when we reached $-62 \mathrm{mV}$

Fig. 1C: It seems to me that the membrane potential is reset after each spike, but details of this reset are missing.

- I precised the reset voltage in the method section.

Fig 1, legend: It is not explained how reference 5 is related to the figure.

- This no longer apply in the current version of the manuscript

It is not clear why CFBP is compact. It is an $n=4$-dimensional problem, so it is defined by its $2^{\wedge} 4=16$ input-output pairs. Even if we restrict ourselves to the mappings with exactly 2 of the inputs being active, there are 6 of such pairs. I understand that it remains linearly non-separable no matter how we define the remaining two mappings, but the definition ('compact because they have four input/output lines') still feels somewhat vague and arbitrary.

- The cFBP is compact because only four input lines need to be defined all the other remaining 12 can take any other value, we now explain this in the result section

The statement 'a reduction in driving force does not generate sublinear summation in a DIF' is false. Reduction of driving force would generate sublinear summation even in a DIF. What the author might want to say is that in this particular example sublinear integration was not associated with reduction of driving force.

- The reduction in driving force would be insufficient in an isopotential neuron as it would affect the independence of g_1 and g_2. I now further underline this point in the discussion.

Last paragraph: It is unclear how the proposed experiment would test whether the granule cells implementing CFBP use saturating input conductance or driving force reduction as a biophysical mechanism to solve the FBP. 
- I agree the experiment would only demonstrate that granule cells are capable of linearly inseparable computation. However, given their isopotential structure it is unlikely that saturation is due to a localized reduction in driving force. This point is now further emphasised in the discussion.

Competing Interests: No

Reviewer Report 13 October 2021

https://doi.org/10.5256/f1000research.77421.r94490

(c) 2021 Papoutsi A et al. This is an open access peer review report distributed under the terms of the Creative Commons Attribution License, which permits unrestricted use, distribution, and reproduction in any medium, provided the original work is properly cited.

\section{Athanasia Papoutsi}

Institute of Molecular Biology \& Biotechnology, Foundation for Research \& Technology - Hellas, Heraklion, Greece

\section{Spyridon Chavlis}

Institute of Molecular Biology \& Biotechnology, Foundation for Research \& Technology, Hellas, Heraklion, Greece

We appreciate the clarifications/corrections made by the author and we support this work for indexing. We have some minor comments that do not change the impact of the work.

Page 5: "You can observe on Figure 1 that locally bounding g enables implement the of cFBP". Consider removing the 'of' preposition.

Figure Legend: "filled circles stand for a $>50 \mathrm{~Hz}$ input spike train while empty circles stand for $>50 \mathrm{~Hz}$ input spike train.", please change the second ">" to "<".

Figure 1B and C: In Figure 1B, the color scheme denotes the LIF vs. DIF models. The same color scheme is used in panel $C$ to denote the scattered vs. clustered case. As it stands now, is confusing. Consider changing the line style on panel $\mathrm{C}$.

Figure Legend: "In the clustered case (grey), the neuron reach spike threshold three times whereas it reaches spike thresold seven times in the scattered case (black)." Please correct "thresold" to "threshold". Also, from the figure, in the in the cluster case it reaches the spike threshold two times (not three).

Please update the Github link to point to the revised code.

There is a mismatch with code and text: code lines 71 and 77: the $\mathrm{g}$ is bounded between 0 and $0.1 \mathrm{nS}$ (i.e., 100pS), while in the text referred to as $10 \mathrm{pS}$. 
Figure 1 Legend: We suggest adding the respective episodes of the truth table in Table 1.

Definition 2: the number of variables denoted with $\mathrm{n}$ should be in italic for consistency.

Is the work clearly and accurately presented and does it cite the current literature?

Yes

Is the study design appropriate and is the work technically sound?

Yes

Are sufficient details of methods and analysis provided to allow replication by others?

Yes

If applicable, is the statistical analysis and its interpretation appropriate?

Yes

Are all the source data underlying the results available to ensure full reproducibility? Yes

Are the conclusions drawn adequately supported by the results?

Yes

Competing Interests: No competing interests were disclosed.

Reviewer Expertise: computational neuroscience; dendritic computations

We confirm that we have read this submission and believe that we have an appropriate level of expertise to confirm that it is of an acceptable scientific standard.

\section{Author Response 01 Feb 2022}

Romain Cazé, CNRS UMR 8520, France

We will respond thoroughly to these minor comments as we receive new review reports. We would like to direct your attention to an important point. This work is based on a strong prediction/hypothesis: that multiple presynaptic neurons target the same postsynaptic receptor, which gives a new role for glutamate spillover.

Competing Interests: No competing interests were disclosed.

\section{Author Response 10 Jun 2022}

Romain Cazé, CNRS UMR 8520, France

Page 5: "You can observe on Figure 1 that locally bounding g enables implement the of CFBP". Consider removing the 'of' preposition. 
Figure Legend: "filled circles stand for $a>50 \mathrm{~Hz}$ input spike train while empty circles stand for $>50 \mathrm{~Hz}$ input spike train.", please change the second ">" to "<".

Definition 2: the number of variables denoted with $\mathrm{n}$ should be in italic for consistency.

- I corrected these mistakes which were introduced during the editorial process

Figure 1B and C: In Figure 1B, the color scheme denotes the LIF vs. DIF models. The same color scheme is used in panel $C$ to denote the scattered vs. clustered case. As it stands now, is confusing. Consider changing the line style on panel $C$.

- I worked on the figure so panel B and C do not conflict we each other anymore

Figure Legend: "In the clustered case (grey ), the neuron reach spike threshold three times whereas it reaches spike thresold seven times in the scattered case (black)." Please correct "thresold" to "threshold". Also, from the figure, in the in the cluster case it reaches the spike threshold two times (not three).

- The panel has now changed and we rewrote the legend.

Please update the Github link to point to the revised code.

There is a mismatch with code and text: code lines 71 and 77: the $g$ is bounded between 0 and $0.1 \mathrm{nS}$ (i.e., 100pS), while in the text referred to as 10ps.

- I updated the git repo and corrected the text to solve this mismatch

Figure 1 Legend: We suggest adding the respective episodes of the truth table in Table 1.

- I followed the reviewer's suggestion.

Competing Interests: No

\section{Version 1}

Reviewer Report 13 July 2021

https://doi.org/10.5256/f1000research.57398.r89096

(C) 2021 Papoutsi A. This is an open access peer review report distributed under the terms of the Creative Commons Attribution License, which permits unrestricted use, distribution, and reproduction in any medium, provided the original work is properly cited. 


\section{Athanasia Papoutsi}

Institute of Molecular Biology \& Biotechnology, Foundation for Research \& Technology - Hellas, Heraklion, Greece

This Brief Report shows at a conceptual level that electrically compact neurons can solve nonlinearly separable computations of four or more inputs. This is a result of the saturating responses to 'clustered' input at the dendritic level (simulating mainly the reduction of the driving force in the dendrites), and increased response to 'scattered' input at the somatic level. This study expands on previous work of the author and others and adds on the range of computations neurons can perform with their dendrites.

We have two major concerns that limit the clarity of this work:

1. Figure 1B and 3rd paragraph on page 5: The $\mathrm{x}$-axis label states 'Time', and the relevant text states that "the membrane voltage takes more time to reach threshold in the clustered case (total $\mathrm{g}=10 \mathrm{pS}$ ) than in the scattered case (total $\mathrm{g}=20 \mathrm{pS}) "$. Given that this work is based on an arbitrary thresholding of the output frequency, it is not obvious where time is involved and its meaning in the $x$-axis.

2. In the provided code on GitHub (line 78 in the code), the ceiling of the second dendrite (i.e., syn 2 in the code) is set to 0.5 and not to 0.1 . Please clarify the value used. If those different saturating thresholds were indeed used, this should be explicitly stated and reasoned in the main text.

Minor comments (not in order of importance nor appearance in the manuscript):

1. For clarity, specify that granule cells refer to the cerebellum (and not the hippocampus).

2. Correct R units to be $M \Omega($ not $m \Omega)$.

3. Figure 1 legend: "filled circles stand for a $>50 \mathrm{~Hz}$ input spike train while empty circles stand for $>50 \mathrm{~Hz}$ input spike train." Change the second $>$ to $<$.

4. Page 4: "This computation that we call the the compact feature binding problem (cFBP) is linearly inseparable." Delete the second 'the'.

Is the work clearly and accurately presented and does it cite the current literature? No

Is the study design appropriate and is the work technically sound?

Yes

Are sufficient details of methods and analysis provided to allow replication by others? Yes

If applicable, is the statistical analysis and its interpretation appropriate? Yes

Are all the source data underlying the results available to ensure full reproducibility? 
Yes

Are the conclusions drawn adequately supported by the results?

Yes

Competing Interests: No competing interests were disclosed.

Reviewer Expertise: computational neuroscience; dendritic computations

I confirm that I have read this submission and believe that I have an appropriate level of expertise to confirm that it is of an acceptable scientific standard, however I have significant reservations, as outlined above.

The benefits of publishing with F1000Research:

- Your article is published within days, with no editorial bias

- You can publish traditional articles, null/negative results, case reports, data notes and more

- The peer review process is transparent and collaborative

- Your article is indexed in PubMed after passing peer review

- Dedicated customer support at every stage

For pre-submission enquiries, contact research@f1000.com

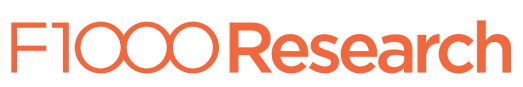

